\title{
Molecular Packing and Photoluminescence Efficiency in Odd-Membered Oligothiophene $S, S$-Dioxides
}

\author{
Luciano Antolini, ${ }^{\dagger}$ Emilio Tedesco, ${ }^{\ddagger}$ Giovanna Barbarella, $*$, Laura Favaretto,, \\ Giovanna Sotgiu, ${ }^{\S}$ Massimo Zambianchi, ${ }^{\S}$ Daniele Casarini,,${ }^{-}$Giuseppe Gigli, ${ }^{\perp}$ and \\ Roberto Cingolani ${ }^{\perp}$
}

\begin{abstract}
Contribution from the Dipartimento di Chimica, Universita' di Modena e Reggio Emilia, Via Campi 183, 41100 Modena, Italy, School of Chemistry, University of Birmingham, Edgbaston, Birmingham B15 2TT, U.K., Consiglio Nazionale Ricerche, I.Co.C.E.A, Via Gobetti 101, 40129 Bologna, Italy, Dipartimento di Chimica, Universita'della Basilicata, via Nazario Sauro 85, 80100 Potenza, Italy, and Istituto Nazionale di Fisica della Materia, Dipartimento Ingegneria Innovazione, Università di Lecce, Via Arnesano, 73100 Lecce, Italy
\end{abstract}

Received March 7, 2000. Revised Manuscript Received May 15, 2000

\begin{abstract}
The single-crystal X-ray structures of three odd-membered thiophene oligomers bearing one central thienyl-S,S-dioxide moiety-trimer, pentamer, and heptamer-are reported. Absolute photoluminescence quantum yields in microcrystalline powders are given for all compounds. The solid-state photoluminescence efficiencies of the trimer $(45 \%)$ and the pentamer $(12 \%)$ were up to 1 order of magnitude higher than those generally measured in conventional oligothiophenes, while that of the heptamer amounted to only $2 \%$. These results are accounted for in terms of molecular packing characteristics, which, owing to the competing effects of dipolar intermolecular interactions between the sulfonyl groups and intra- and intermolecular $\mathrm{C}-\mathrm{H} \cdots \mathrm{O}$ hydrogen bondings and $S \cdots S$ interactions, change dramatically on changing the oligomer size. While the trimer is highly distorted and crystallizes in a chiral orthorhombic space group with the molecular long axes markedly tilted with respect to one another, the heptamer displays a coplanar conformation with the molecules packing in strictly parallel layers. Contrary to the solid state, the photoluminescence intensity in solution increases on increasing the oligomer size and shows marked solvent dependence.
\end{abstract}

The increasing demand of new organic molecular materials with exciting electrical and optical characteristics for device fabrication ${ }^{1}$ requires the elucidation of the relationship existing between molecular structure and solid-state properties. This means designing molecular systems capable of organizing according to a well-defined supramolecular architecture in the solid state that can be related to a given electrical and/or optical property. However, generally, the solid-state properties of molecular aggregates are the result of the interplay of different subtle factors which are difficult to separate and identify. Even a slight structural modification of a given molecule may have a great effect on its capacity to self-assemble and lead to

\footnotetext{
* Corresponding author. E-mail: barbarella@area.bo.cnr.it.

†Universita' di Modena e Reggio Emilia.

$\div$ University of Birmingham.

$\S$ Consiglio Nazionale Ricerche.

"Universita' della Basilicata.

$\perp$ Università di Lecce.

(1) Tang, C. W.; VanSlyke, S. A. Appl. Phys. Lett. 1987, 51, 913. (b) Burroughes, J. H.; Bradley, D. D. C.; Brown, A. R.; Marks, R. N.; Mackay, K.; Friend, R. H.; Burn, P. L.; Holmes, A. B. Nature 1990, 347, 539. (c) Berggren, M.; Inganäs, O.; Gustafsson, G.; Rasmusson, J.; Andersson, M. R.; Hjertberg, T.; Wennerström, O. Nature 1994, 372, 444. (d) Sheats, J. R.; Antoniadis, H.; Hueschen, M.; Leonard, W.; Miller, J.; Moon, R.; Roitman, D.; Stocking, A. Science 1996, 273, 884. (e) Cao, Y.; Parker, I. D.; Yu, G.; Zhang, C.; Heeger, A. J. Nature 1999, 397, 414. (f) Leising, G.; Tasch, S.; Graupner, W. In Handbook of conductive polymers; Skotheim, T. A., Elsenbaumer, R. L., Reynolds, J. R., Eds.; Marcel Dekker: New York, 1998; p 847. (g) Burrows, P. E.; Bulovic, V.; Gu, G.; Kozlov, V.; Forrest, S. R.; Thompson, M. E. Thin Solid Films 1998, 331, 101. (h) Deshpande, R. S.; Bulovic, V.; Forrest, S. R. Appl. Phys. Lett. 1999, 75, 888. (i) O'Brien, D. F.; Baldo, M. A.; Thompson, M. E.; Forrest. S. R. Appl. Phys. Lett. 1999, 74, 442.
}

unpredictable collective behavior in regard to the properties of interest. Thus, the rational design of new organic molecular materials requires extensive experimental and theoretical investigations on the way they self-assemble in the solid state.

Conventional thiophene oligomers, namely those with no substituents at the thienyl sulfur, generally display very low photoluminescence quantum yields in the solid state. ${ }^{2}$ Functionalization with alkyl groups is insufficient to ensure the increase in the solid-state photoluminescence efficiency; however, further functionalization of one thienyl sulfur with oxygen - namely the transformation of one thienyl moiety to the corresponding thienyl-S,S-dioxide ${ }^{3}$-may lead to an amazing increase in this parameter. ${ }^{4 a}$ High solid-state photoluminescence efficiencies combined with high electron affinities-also brought about by the presence of the thienyl-S,S-dioxide units ${ }^{4 \mathrm{~b}}$ - has allowed the fabrication of highly efficient electroluminescent

(2) (a) Ziegler, C. In Handbook of organic conductive molecules and polymers; Nalwa, H. S., Ed.; John Wiley \& Sons Ltd.: New York, 1997; p 677. (b) Väterlein, C.; Neureiter, H.; Gebauer, W.; Ziegler, B.; Sokolowski, M.; Bäuerle, P.; Umbach, E. J. Appl. Phys. 1997, 82, 3003. (c) Kouki, F.; Spearman, P.; Horowitz, G.; Delannoy, P.; Valat, P.; Wintgens, V.; Garnier, F. Synth. Met. 1999, 102, 1071.

(3) (a) Gronowitz, S. Phosphorus, Sulfur Silicon 1993, 74, 113. (b) Nakayama, J.; Nagasawa, H.; Sugihara, Y.; Ishii, A. J. Am. Chem. Soc. 1997, 119, 9077. (c) Barbarella, G.; Favaretto, L.; Sotgiu, G.; Zambianchi, M.; Antolini, L.; Pudova, O.; Bongini, J. Org. Chem. 1998, 63, 5497.

(4) (a) Barbarella, G.; Favaretto, L.; Sotgiu, G.; Zambianchi, M.; Fattori, V.; Cocchi, M.; Cacialli, F.; Gigli, G.; Cingolani, R. Adv. Mater. 1999, 11, 1375. (b) Barbarella, G.; Favaretto, L.; Zambianchi, M.; Pudova, O.; Arbizzani, C.; Bongini, A.; Mastragostino, M. Adv. Mater. 1998, 10, 551. (c) Gigli, G.; Barbarella, G.; Favaretto, L.; Cacialli, F.; Cingolani. R. Appl. Phys. Lett. 1999, 75, 439. 
Table 1. Torsion Angles ( $\phi$ ), Packing Coefficients (PC), Packing Potential Energies (ppe), Molecular Volumes $\left(V_{\mathrm{mol}}\right)$ and Short Intermolecular Contacts of $\mathbf{1 - 3}$

\begin{tabular}{llll}
\hline & \multicolumn{1}{c}{$\mathbf{1}$} & \multicolumn{1}{c}{$\mathbf{2}$} & \multicolumn{1}{c}{$\mathbf{3}$} \\
\hline crystal system & orthorhombic & monoclinic & triclinic \\
space group & $P 2{ }_{1} 2_{1}$ & $P 2_{1} / n$ & $P 1$ \\
asymmetric unit & 1 & 1 & 1 \\
$\quad$ contents (molecules) & & & \\
$\phi_{1}(\mathrm{deg})$ & & & $-7.8(6)$ \\
$\phi_{2}(\mathrm{deg})$ & & $-165.3(3)$ & $178.0(3)$ \\
$\phi_{3}(\mathrm{deg})$ & $-168.4(4)$ & $-171.9(3)$ & $-178.5(2)$ \\
$\phi_{4}(\mathrm{deg})$ & $28.5(8)$ & $-168.5(3)$ & $-174.9(2)$ \\
$\phi_{5}(\mathrm{deg})$ & $-151.6(5)^{a}$ & & \\
& & $-171.2(4)$ & $178.9(2)$ \\
$\phi_{6}(\mathrm{deg})$ & & $15.6(9)^{a}$ & \\
& & & $-3.4(7)$ \\
$V_{\text {mol }}\left(\AA^{3}\right)$ & 391.6 & 515.8 & $-177.7(4)^{a}$ \\
$\mathrm{PC}$ & 0.636 & 0.676 & 0.681 \\
$\mathrm{ppe}\left(\mathrm{kcal} \cdot \mathrm{mol}{ }^{-1}\right)$ & -87.6 & -124.6 & -165.0 \\
$\mathrm{H} \cdots \mathrm{O}(\AA)$ & 2.638 & 2.474 & 2.318 \\
& 2.333 & 2.415 & 2.576 \\
$\mathrm{C}-\mathrm{H} \cdots \mathrm{O}(\mathrm{deg})$ & 164.2 & 152.01 & 141.40 \\
& & 166.75 & 161.75 \\
$\mathrm{~S} \cdots \mathrm{S}(\AA)$ & & 152.55 & \\
& & & 3.253 (intra) \\
& & & 3.323 (intra) \\
\hline
\end{tabular}

${ }^{a}$ Minor conformer.

diodes based on polyalkylated quinquethiophene $S, S$-dioxides. ${ }^{4 a, c}$ Since these results open the way to the use of thiophene oligomers as active emitting materials in the fabrication of lightemitting diodes (LEDs), we have undertaken a study aimed at understanding how the supramolecular organization in the solid state affects the efficiency of photoluminescence in these modified thiophene oligomers. No data have been reported as yet on this subject. Nevertheless, information on this point is crucial to tailor and eventually optimize the luminescence efficiency by altering the molecular packing modalities.

As a first contribution to address the question, the present paper reports the single-crystal X-ray structures of a trimer, pentamer, and heptamer $(\mathbf{1}-\mathbf{3}, \mathrm{R}=$ hexyl):

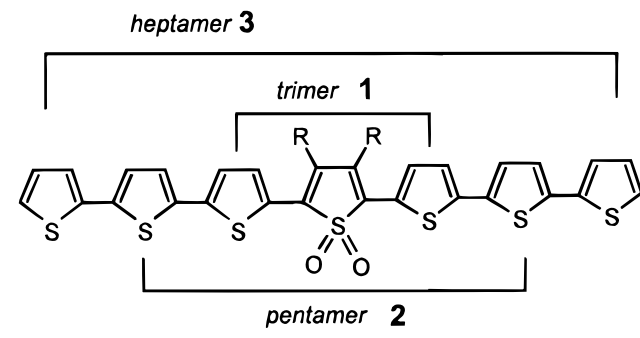

Compounds 1-3 have an odd number of rings and the same pseudosymmetry and differ only in the amount of thienyl groups adjacent to the central thienyl- $S, S$-dioxide moiety. We will show that the competing effects of dipolar interactions, weak hydrogen bondings, and van der Waals interactions result in very different packing modalities on changing the oligomer length and dramatically effect the solid-state photoluminescence efficiencies.

\section{Results}

(I) Solid-State Conformation and Molecular Packing. ORTEP drawings of the molecular structure and crystal packing of compounds $\mathbf{1 - 3}$ are presented in Figures 1-3, respectively. Table 1 summarizes the most relevant structural features of $\mathbf{1}-\mathbf{3}$.

All strucures contain one crystallographically independent molecule and are conformationally disordered. In all the compounds, one of the outer thienyl rings assumes two opposite orientations (anti or syn with respect to the $\mathrm{S}$ atoms of adjacent rings) arising from a rotation of $\sim 180^{\circ}$ around the $\mathrm{C}-\mathrm{C}$ interring bond. Furthermore, in all the compounds, one of the two hexyl chains is affected by conformational disorder involving mainly its fifth $\mathrm{C}$ atom which was found to be distributed over at least two sites. There is good agreement between corresponding bond distances and angles, and their values compare well with those previously observed in many oligothiophenes. ${ }^{5,6}$ In all the compounds, the central thienyl- $S, S$-dioxide ring shows the lengthening of $\mathrm{C}-\mathrm{S}$ (mean value 1.773(7) $\AA$ ) and $\mathrm{C} 3-\mathrm{C} 4$ (mean value $1.480(9) \AA$ ) bonds, both $\sim 0.004 \AA$ longer than the corresponding distances in inner thiophene rings. ${ }^{5} \mathrm{~A}$ parallel shortening of $\mathrm{C} 2-\mathrm{C} 3$ and $\mathrm{C} 4-\mathrm{C} 5$ bonds (mean value 1.356(8) A) was also observed.

The conformation of trimer $\mathbf{1}$ is characterized by $\mathrm{S}-\mathrm{C}-\mathrm{C}-\mathrm{S}$ inter-ring torsion angles of $-168.4(4)$ and $28.5(8)^{\circ}$ or, due to disorder, $-151.6(5)^{\circ}$. The slightly preferred conformation $(51.6 \%)$ is the anti-anti one. The deviations from coplanarity between rings become considerably smaller in pentamer $\mathbf{2}$, whose inter-ring torsion angles range from -165.3(3) to $-171.9(3)^{\circ}$. All ring junctions are of the anti type in the prevailing orientation $(66.2 \%)$ for the disordered outer ring. In heptamer $\mathbf{3}$, the increase in the number of thienyl units leads to a much more planar structure in which the dihedral angles between ring planes range from $1.4(2)$ to $7.3(2)^{\circ}$. The maximum atomic deviations from the mean plane through the whole aromatic backbone is only $0.162 \AA$. Furthermore, the conformation of $\mathbf{3}$ shows a new and interesting feature. Indeed, both outer thienyl rings (in the prevailing conformation, $73.8 \%$ for the disordered one) exhibit syn orientation with respect to adjacent rings and $\mathrm{S}-\mathrm{C}-\mathrm{C}-\mathrm{S}$ torsional angles of $-7.8(6)$ and $3.4(7)^{\circ}$, respectively. To our knowledge, this is the first case of syn orientation between unsubstituted thiophene rings with full or major site occupancy in oligothienyls of known structure. Previously, such a conformation was observed only in molecules affected by rotational disorder for the less populated conformer (never exceeding 20-25\%). ${ }^{5 \mathrm{~b}, \mathrm{~d}}$

As mentioned above, in $\mathbf{1 - 3}$ one of the two hexyl chains is conformationally disordered. It is worth noting that, in the prevailing conformation, all the disordered chains adopt a transgauche-gauche-trans orientation. The hexyl groups not affected by disorder display all-trans orientation in trimer $\mathbf{1}$ and transgauche-trans-trans orientation in both pentamer $\mathbf{2}$ and heptamer 3. In the latter compounds, the two hexyl groups lie on the same side with respect to the mean plane passing through the aromatic backbone, while in $\mathbf{1}$ they are on opposite sides.

In our opinion, the most interesting feature of the molecular packing of trimer $\mathbf{1}$ appears to be the fact that the molecules pack in the crystal in chiral form, thus reaching spontaneous resolution. To our knowledge, among oligothienyls, only $3,4^{\prime}, 4^{\prime \prime}$ -

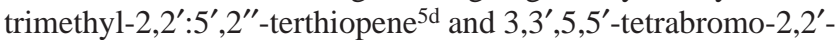
bithiophene $\mathrm{e}^{5 \mathrm{c}}$ have been found to give chiral crystals. The crystal packing of 1 (Figure 1) leads to marked conformational distortions from coplanarity and to the lack of short intermolecular contacts. There is only one van der Waals distance

(5) (a) Antolini, L.; Folli, U.; Goldoni, F.; Mucci, A.; Schenetti. L. Acta Polym. 1998, 49, 248. (b) Barbarella, G.; Zambianchi, M.; Bongini, A.; Antolini, L. Adv. Mater. 1993, 5, 834. (c) Barbarella, G.; Zambianchi, M.; Antolini, L.; Ostoja, P.; Maccagnani, P. Bongini, A.; Marseglia, E. A.; Tedesco, E.; Gigli, G.; Cingolani, R. J. Am. Chem. Soc. 1999, 121, 8920. (d) Barbarella, G.; Zambianchi, M.; Bongini, A.; Antolini, L. Adv. Mater. 1994, 6, 561 .

(6) Porzio, W.; Destri, S.; Mascherpa, M.; Bruckner, S. Acta Polym. 1993, 44,266 

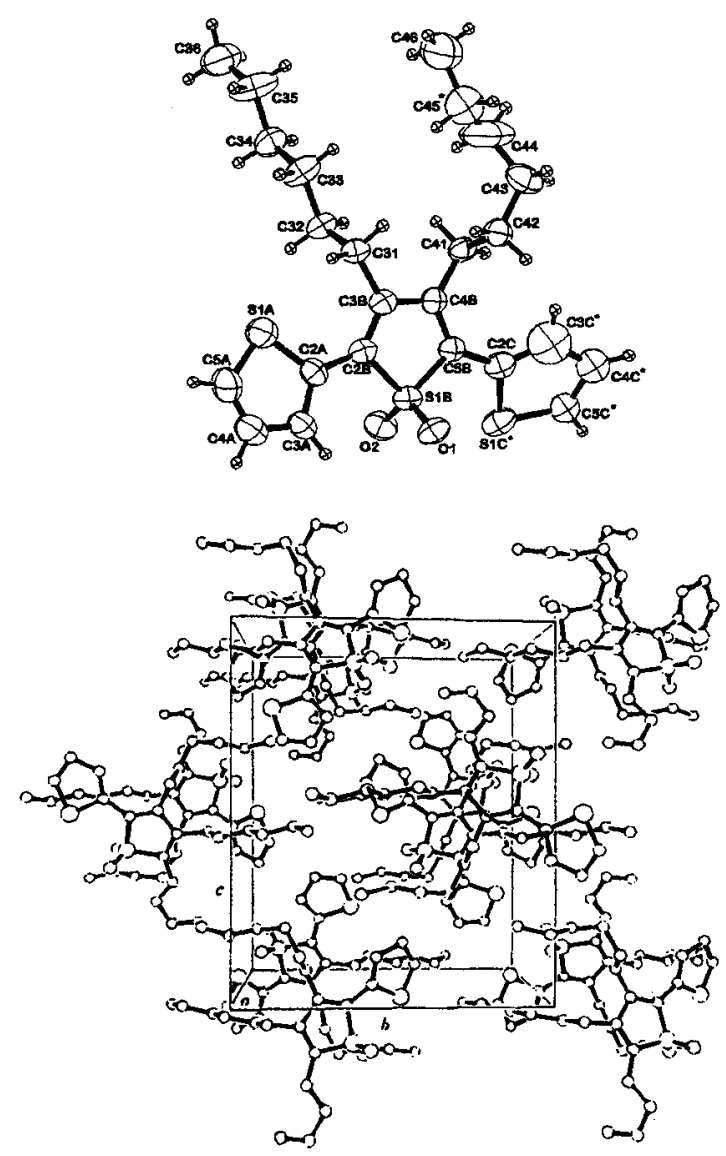

Figure 1. Molecular structure and perspective view of the crystal packing of trimer $\mathbf{1}$.

shorter than $3.60 \AA$, and the shortest $\mathrm{S} \cdots \mathrm{S}$ intermolecular separation is 4.799(3) $\AA$.

In pentamer 2, despite the strong out-of-plane displacements of the oxygen atoms of the sulfonyl group and of the hexyl chains, the molecular packing (Figure 2) is close to the "herringbone" packing motif typical of planar or quasi-planar oligothienyls. Nevertheless, the dihedral angle between the mean planes through aromatic moieties related by glide plane ("herringbone" angle) is $38.51(7)^{\circ}$, thus outside the $55-70^{\circ}$ range normally observed in oligothiophenes. ${ }^{6,7}$ Unlike 1 , the molecular packing of $\mathbf{2}$ is characterized by a number of very short van der Waals contacts, quite rarely observed in oligothiophenes. ${ }^{5}$ The shortest ones occur between thienyl carbons (3.341(8) $\AA$ ) or between thienyl $\mathrm{C}$ and sulfonic $\mathrm{O}$ atoms (3.393(7) $\AA$ ). Somewhat short intermolecular S $\cdots$ S separations (3.741(4) $\AA$ ) are also present in the crystal packing.

Heptamer $\mathbf{3}$ displays a packing mode quite different from that of pentamer 2. Its longer and more planar molecules stack roughly perpendicular to the $b$ cell axis (Figure 3), in exactly parallel layers related by a crystallographic inversion center. The long interlayer distance and, more importantly, a scarce molecular overlap do not allow $\pi-\pi$ interactions to occur between stacked thienyl rings. Nevertheless, a lot of short $\mathrm{S} \cdots \mathrm{O}, \mathrm{S} \cdots \mathrm{C}, \mathrm{C} \cdots \mathrm{O}$, and $\mathrm{C} \cdots \mathrm{C}$ intermolecular contacts, stronger than those observed in $\mathbf{2}$, characterize the crystal packing. This holds also for $\mathrm{S} \cdots \mathrm{S}$ interactions, three of them being shorter than $3.8 \AA$.

Table 1 shows the packing coefficients (PC) and packing potential energies (ppe) of $\mathbf{1}-\mathbf{3}$ estimated using the atom-atom potential energy method. ${ }^{8 \mathrm{a}, \mathrm{b}}$ The crystal packing analysis was made difficult by the presence of crystallographic disorder, which affected the terminal thienyl rings and some carbon atoms
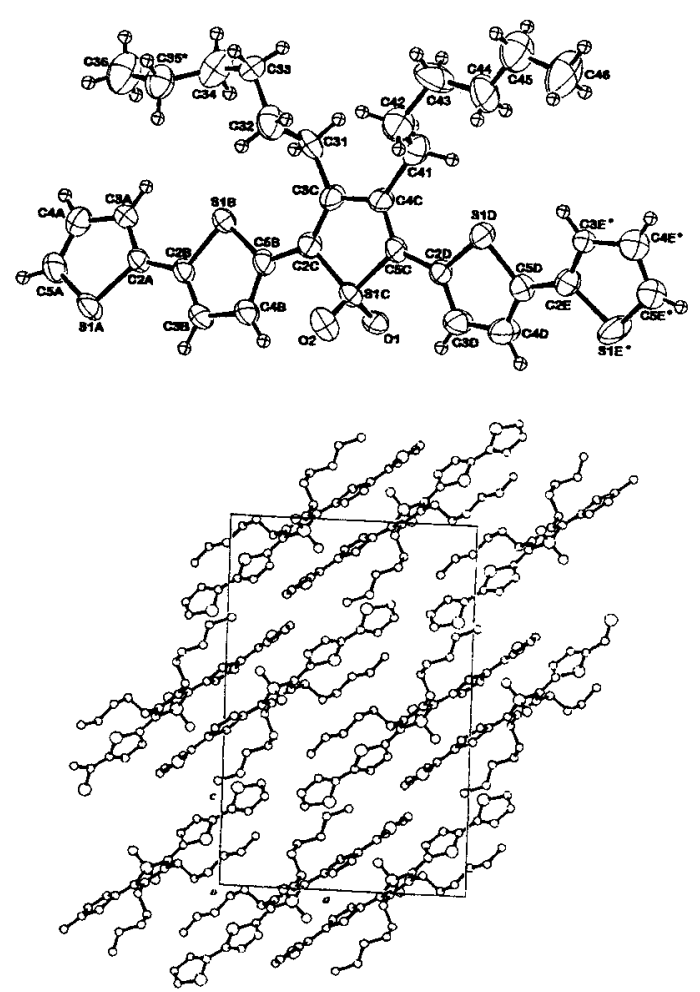

Figure 2. Molecular structure and projection of the crystal packing down $b$ cell axis of pentamer 2 .
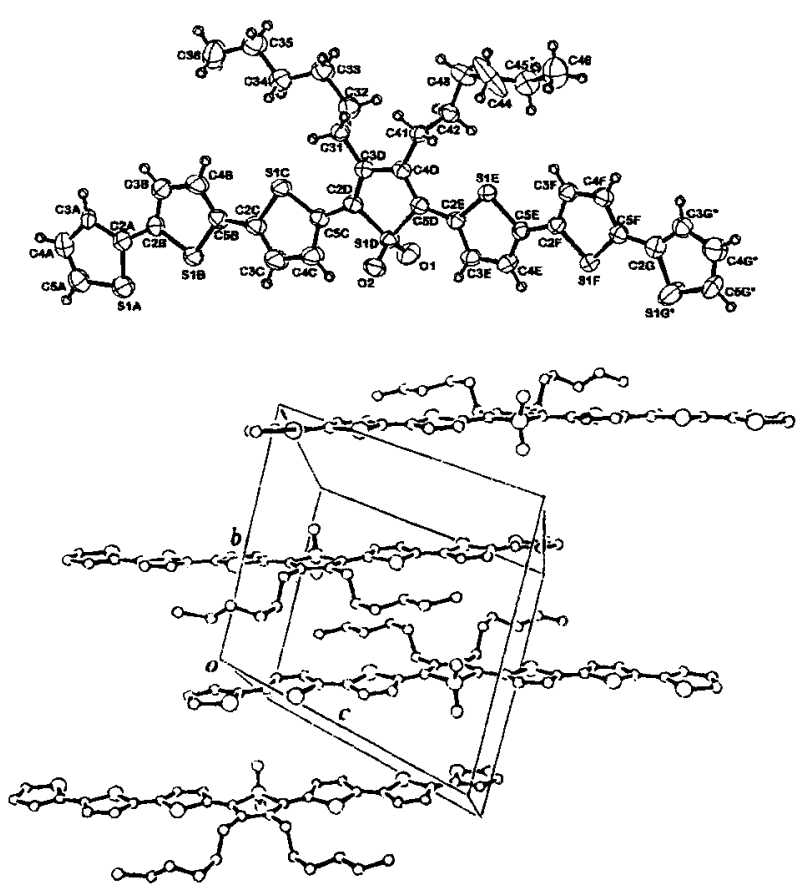

Figure 3. Molecular structure and perspective view of the crystal packing of heptamer 3 .

of the hexyl chains. Thus, the calculations were made by considering all the possible "ordered" crystals of these compounds.

There is obviously an interplay between the molecular structure and the overall crystal packing of these oligomers. As

(7) Antolini, L.; Horowitz, G.; Kouki, F.; Garnier, F. Adv. Mater. 1998, 10,385 .

(8) (a) Persin, A. J.; Kitaigorodsky, A. I. The Atom-Atom Potential Method; Springer-Verlag: Berlin, 1987. (b) Gavezzotti, A. J. Am. Chem. Soc. 1983, 105, 5220. 

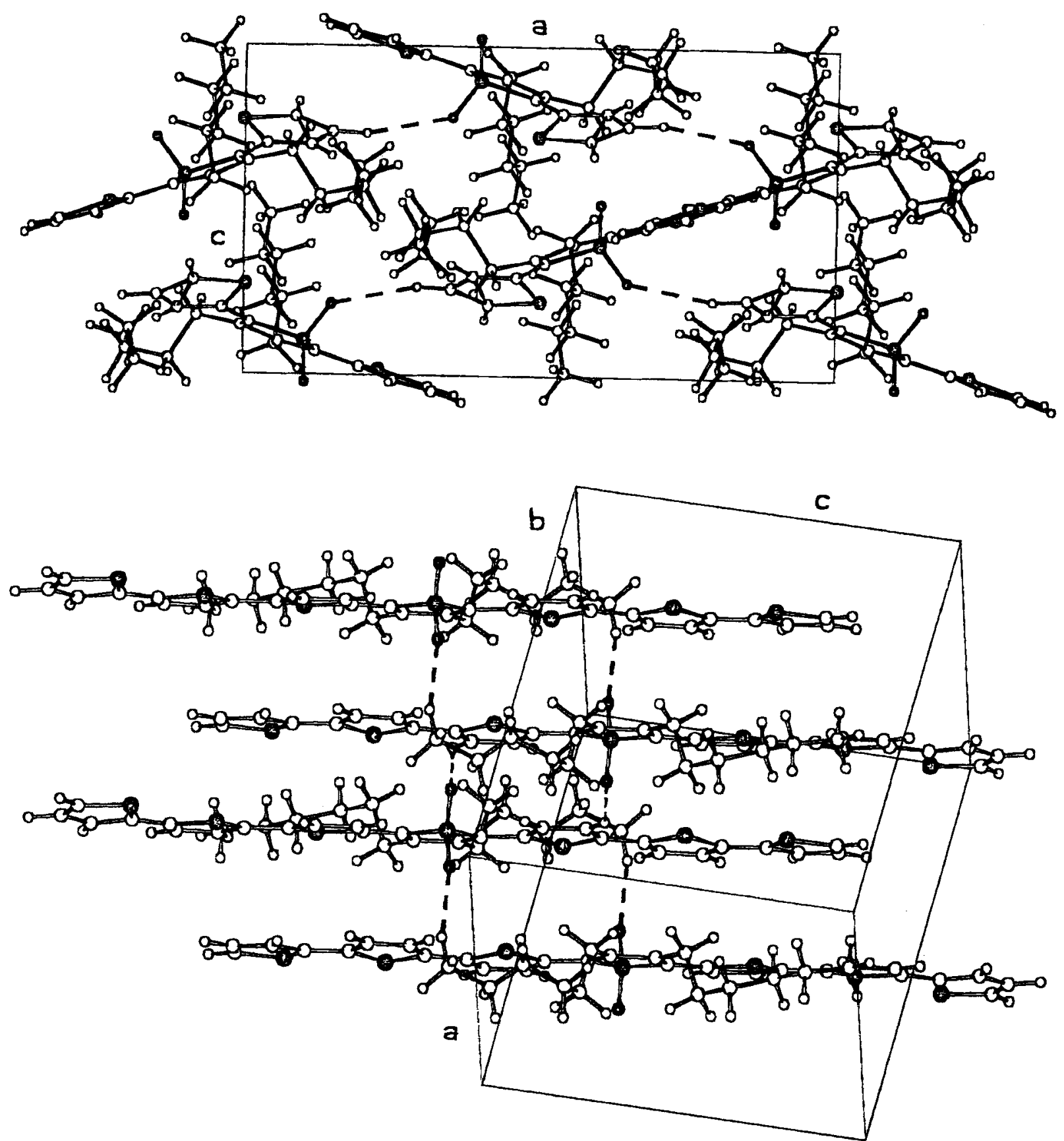

Figure 4. Top: view along the $b$ cell axis of the intermolecular $\mathrm{C}-\mathrm{H} \cdots \mathrm{O}$ hydrogen bondings $(2.638 \AA$, dashed lines) of trimer 1 . Bottom: layers of molecules of heptamer 3 interacting via short $\mathrm{C}-\mathrm{H} \cdots \mathrm{O}$ intermolecular contacts (dashed lines, see Table 1).

gathered from the PC values in Table 1, the crystal density increases on going from $1(0.636)$ to $3(0.681)$, showing that the packing efficiency is achieved more easily by planar conformations ${ }^{9}$ as already observed for conventional oligothiophenes. ${ }^{10}$ There are several intermolecular interactions that must be optimized in these systems. For instance, the longrange dipole-dipole interactions due to the presence of the $\mathrm{SO}_{2}$ groups drive the molecules of $\mathbf{1 - 3}$ to align in an antiparallel fashion in the solid-state. Intermolecular $\mathrm{C}-\mathrm{H} \cdots \mathrm{O}$ hydrogen bonds become shorter on going from $\mathbf{1}$ to $\mathbf{3}$ (see Table 1) since the increased number of thienyl rings allows a closer approach of "acidic" thienyl hydrogens toward the oxygen atoms. Accordingly, we find for heptamer 3 the shortest $\mathrm{C}-\mathrm{H} \cdots \mathrm{O}$

(9) Desiraju, G. R. Acc. Chem. Res. 1991, 24, 290.

(10) Marseglia, E. A.; Grepioni, F.; Tedesco, E.; Braga, D. Mol. Cryst. Liq. Cryst., in press. hydrogen bond in this series $(2.318 \AA)$. The trend is illustrated in Figure 4, which shows the crystal arrangement of the molecules of 1 along the $b$-axis and forming $\mathrm{C}-\mathrm{H} \cdots \mathrm{O}$ hydrogen bondings of $2.638 \AA$ and layers of molecules of $\mathbf{3}$ interacting via short $\mathrm{C}-\mathrm{H} \cdots \mathrm{O}$ intermolecular contacts.

Furthermore, the role played by the hexyl chain grafted at the $\beta$-position should not be neglected in these compounds. As observed above, the more distorted hexyl moieties allow molecules of $\mathbf{1}$ to stay "far apart" from each other (see Figure 1), while in the case of $\mathbf{2}$ and $\mathbf{3}$, the alkyl groups are easily accommodated into the solid-state arrangement and do not hamper short intermolecular interactions (see Figures 2 and 3, respectively).

(II) ${ }^{13}$ C CP-MAS NMR. We have already shown that carbon13 cross-polarization magic angle spinning nuclear magnetic resonance $\left({ }^{13} \mathrm{C} \mathrm{CP}-\mathrm{MAS} \mathrm{NMR}\right)$ of thiophene oligomers may 

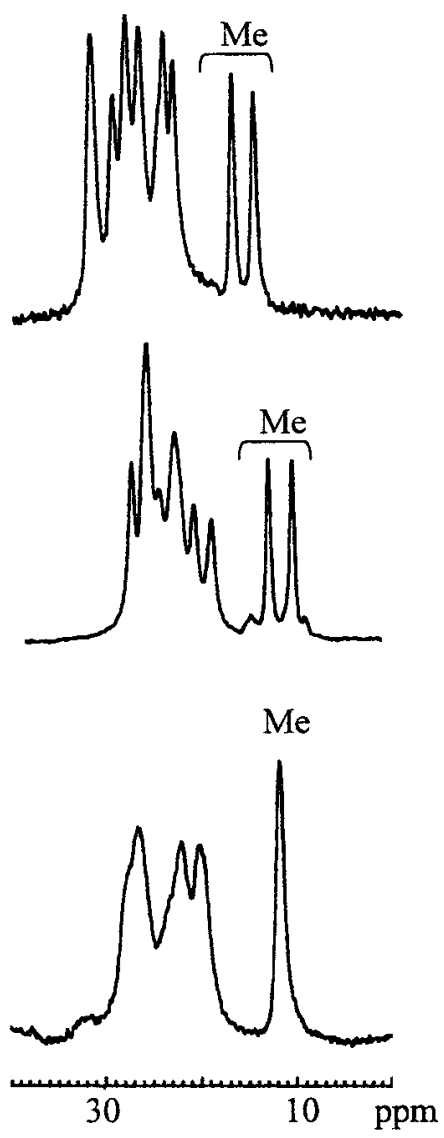

Figure 5. Aliphatic region of the ${ }^{13} \mathrm{C}$ CP-MAS NMR spectrum of the microcrystalline powders of $\mathbf{1}-\mathbf{3}$.

furnish useful information on their solid-state short-range organization properties. ${ }^{11} \mathrm{NMR}$ is very sensitive to the local chemical environment of molecules and to conformational changes induced by different packing types. ${ }^{11}$ However, to date, only very few ${ }^{13} \mathrm{C}$ CP-MAS NMR studies have been carried out on this kind of compound and comparison with single-crystal $\mathrm{X}$-ray data is still needed to verify the reliability of the technique. Thus, we felt justified in reporting here some information on the ${ }^{13} \mathrm{C}$ CP-MAS NMR spectra of the microcrystalline powders of 1-3 which furnish a useful test since in $\mathbf{1}$ and $\mathbf{2}$ the aliphatic chains are in chemically and magnetically different environments while in $\mathbf{3}$ the aliphatic chains become magnetically equivalent due to the rigorously planar conformation (see Figures 1-3).

Figure 5 shows the aliphatic region of the ${ }^{13} \mathrm{C}$ CP-MAS NMR spectrum of $\mathbf{1 - 3}$. The figure shows that there are two different signals for the terminal methyl groups of the hexyl chains of $\mathbf{1}$ $\left(\delta_{\mathrm{TMS}}=15.1\right.$ and $\left.17.3 \mathrm{ppm}\right)$ and $\mathbf{2}\left(\delta_{\mathrm{TMS}}=14.3\right.$ and $\left.16.7 \mathrm{ppm}\right)$. On the contrary, only one single signal is observed for the same groups in heptamer $3\left(\delta_{\mathrm{TMS}}=15.8 \mathrm{ppm}\right)$. Thus, solid-state carbon-13 NMR data on the microcrystalline powders of 1-3 fit nicely with the conformational information obtained from single-crystal X-ray diffraction. This result confirms that the ${ }^{13} \mathrm{C}$ CP-MAS NMR technique is a reliable alternative to get solid-state conformational information when single crystals of thiophene-based oligomers cannot be obtained.

(III) Photoluminescence in the Solid State and in Solution. Table 2 shows the maximum absorption and photoluminescence (PL) wavelengths of $\mathbf{1 - 3}$ in solution and PL wavelengths and

(11) Barbarella, G.; Casarini, D.; Zambianchi, M.; Favaretto, L.; Rossini, S. Adv. Mater. 1996, 8, 69.
Table 2. Maximum Wavelength Absorption $\left(\lambda_{\max }\right)$ and Emission $\left(\lambda_{\mathrm{PL}, \mathrm{sol}}\right)$ Values of $\mathbf{1 - 3}$ in Solution ${ }^{a}$ and Photoluminescence Frequencies $\left(\lambda_{\mathrm{PL}, \text { powd }}\right)^{b}$ and Efficiencies $(\eta)$ of the Same Compounds as Microcrystalline Powders

\begin{tabular}{ccccc}
\hline compd & $\lambda_{\max }{ }^{a}(\mathrm{~nm})$ & $\lambda_{\mathrm{PL}, \text { sol }}{ }^{a}(\mathrm{~nm})$ & $\lambda_{\mathrm{PL}, \text { powd }}{ }^{c}(\mathrm{~nm})$ & $\eta^{d}(\%)$ \\
\hline $\mathbf{1}$ & 409 & 520 & 530 & 45 \\
$\mathbf{2}$ & 475 & 590 & 665 & 12 \\
$\mathbf{3}$ & 495 & 620 & 725 & 2 \\
\hline
\end{tabular}

${ }^{a}$ In $\mathrm{CH}_{2} \mathrm{Cl}_{2}$ and Decalin, $10^{-5} \mathrm{M}$ solutions. ${ }^{b}$ Under UV excitation. ${ }^{c}$ Microcrystalline powders. ${ }^{d}$ Absolute values obtained for the microcrystalline powders by using an integrating sphere.

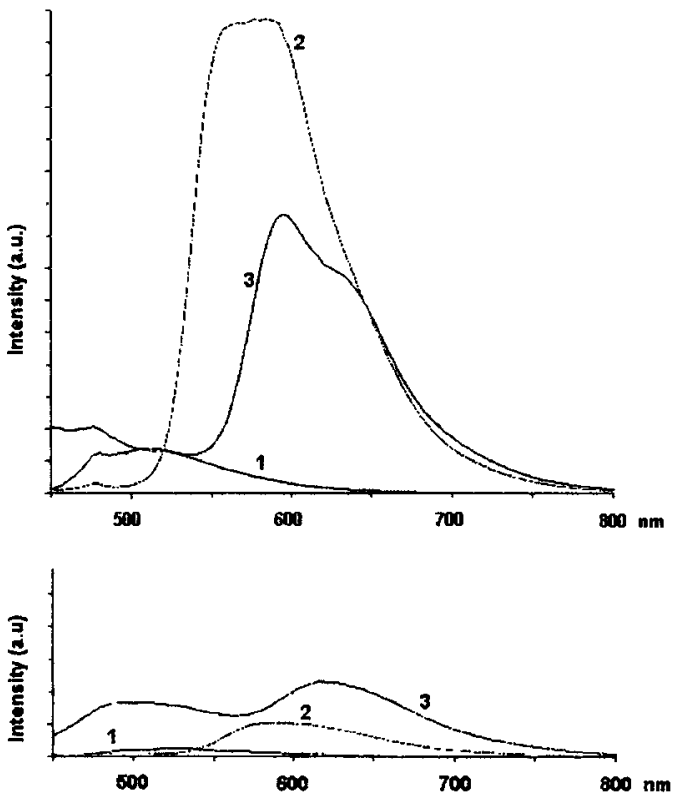

Figure 6. Photoluminescence spectra of $1-310^{-5} \mathrm{M}$ in methylene chloride (bottom) and Decalin (top).

efficiencies of the same compounds as microcrystalline powders. Figure 6 shows the PL spectra of $1-310^{-5} \mathrm{M}$ in methylene chloride and Decalin.

As expected, the maximum wavelength absorption, $\lambda_{\max }$, increases on increasing the oligomer size, owing to increasing $\pi-\pi$ delocalization.

The PL wavelength in solution, $\lambda_{\text {PLsol }}$, also increases on going from 1 to 3 and displays a Stokes shift of $\sim 0.5 \mathrm{eV}$, i.e., of the same order as that observed for conventional oligothiophenes. ${ }^{12}$ The wavelengths of $\mathbf{1 - 3}$ in the solid state are all red shifted with respect to those in solution. The red shift is much greater for the longer oligomers $2(0.24 \mathrm{eV}, 75 \mathrm{~nm})$ and $3(0.29 \mathrm{eV}$, $105 \mathrm{~nm})$ than for the shorter one (1, $0.05 \mathrm{eV}, 10 \mathrm{~nm})$. Similar red shifts have been observed for conventional thiophene oligomers and may be the results of several effects ${ }^{13}$ including conformational factors, i.e., greater backbone distortions in solution with respect to the solid state. ${ }^{5 \mathrm{~b}, \mathrm{~d}, 13}$

Table 2 shows that, in the solid state, the PL quantum yield decreases on increasing the oligomer size and varies from $45 \%$ in trimer $\mathbf{1}$ to $12 \%$ in pentamer $\mathbf{2}$ and $2 \%$ in heptamer $\mathbf{3}$. Preliminary measurements of time-resolved solid-state photoluminescence of $\mathbf{1}$ to $\mathbf{3}$ indicate that on going from the trimer to the hexamer there is a small variation in radiative decay times $\left(\tau_{\mathrm{R}}\right)$ while there is a dramatic change in nonradiative ones $\left(\tau_{\mathrm{NR}}\right)$. For example, $\tau_{\mathrm{R}}$ is 3171 and $3000 \mathrm{~ns}$ for $\mathbf{1}$ and $\mathbf{3}$, respectively,

(12) (a) Chosrovian, H.; Rentsch, S.; Grebner, D.; Dahm, U.; Birckner E. Synth. Met. 1993, 60, 23. (b) Kanemitsu, Y.; Suzuki, K.; Masumoto, Y.; Tomiuchi, Y.; Shiraishi, Y.; Kuroda. M. Phys. Rev. B 1994, 50, 2301.

(13) Yang, A.; Kuroda, M.; Shiraishi, Y.; Kobayashi, T. J. Chem. Phys. 1998, 109,8442 . 
while $\tau_{\mathrm{NR}}$ is 2594 and $61 \mathrm{~ns}$. A detailed study of time-resolved solid-state photoluminescence of oligothiophene $S, S$-dioxides will be reported in a forthcoming paper.

The PL spectra of $\mathbf{1 - 3}$ in $\mathrm{CH}_{2} \mathrm{Cl}_{2}$ reported in Figure 6 (bottom) show a very low intensity for all the compounds, including trimer $\mathbf{1}$. However, on changing the solvent from $\mathrm{CH}_{2} \mathrm{Cl}_{2}$ to Decalin, a remarkable increase of almost 1 order of magnitude (without any change of the PL emission wavelengths) is observed for the intensity of the longer oligomers $\mathbf{2}$ and $\mathbf{3}$, whereas the PL intensity of $\mathbf{1}$ is scarcely affected (Figure 6, top).

Measuring the PL quantum yields of $\mathbf{1 - 3}$ in solution was beyond the aim of the present work. For pentamer $\mathbf{2}$ in the same solvent, we have already reported a PL quantum yield of only $0.5 \%,{ }^{4 a}$ a value which was much lower than that measured for unsubstituted quinquethiophene $(\eta=40 \%)$ and for its dihexyl derivative $(\eta=9 \%)$ precursors of pentamer $2 .{ }^{4 a}$ The sound comparison of the photoluminescence efficiencies of $\mathbf{1 - 3}$ in solution with those in the solid state would require at least a rough estimate of the conformational changes in the two environments, since conformational changes affect the electronic properties. The electronic properties, in turn, affect the balance between radiative and nonradiative pathways for energy relaxation from the excited state. ${ }^{12}$

Nevertheless, the spectra reported in Figure 6 indicate unambiguously that photoluminescence intensity of $\mathbf{1 - 3}$ is greatly dependent on the nature of the solvent, contrary to what is generally observed in conventional oligothiophenes. ${ }^{12 a}$ When Decalin is used, the photoluminescence intensity of the longer and conformationally more mobile oligomers $\mathbf{2}$ and $\mathbf{3}$ is 1 order of magnitude greater than that in methylene chloride. Moreover, the trend of photoluminescence intensity on increasing the oligomer size approaches that observed for conventional oligothiophenes. $^{12}$

At the present state of knowledge, it is difficult to understand whether it is the difference in the dielectric constant or the viscosity or in the other characteristics of the two solvents that is responsible for the changes in the photoluminescence intensity. Compared to conventional oligothiophenes, oligothiophene $S, S$-dioxides, characterized by the presence of a strongly polar sulfonyl group, are likely to be much more susceptible to solvent variations. Moreover, in these amphiphilic compounds, polar solvents should tend to solvate the molecules at the hydrophilic end, while nonpolar solvents should tend to interact more through the aliphatic chains. In any case, solute-solvent interactions should furnish more efficient nonradiative energy relaxation pathways from the excited state than in conventional oligothiophenes.

The fact that the photoluminescence intensity of trimer $\mathbf{1}$ is scarcely affected by the change in solvent seems to indicate that conformational rigidity plays an important role in determining the photoluminescence properties of oligothiophene $S, S$ dioxides. In the ground state, the conformation of $\mathbf{1}$ is likely to be rather rigid, owing to the energy barriers to rotation around the $\mathrm{C}-\mathrm{C}$ bonds linking the thienyl-S,S-dioxide moiety to the adjacent thienyl ring. ${ }^{3 \mathrm{c}}$ Probably, some of this conformational rigidity is transferred also to the excited state, which has greater quinoidic character.

\section{Discussion}

The light emission properties of thiophene oligomers and polymers are far from being fully understood despite great improvements in the past few years. ${ }^{14}$ Most conventional thiophene oligomers display solid-state photoluminescence efficiencies on the order of $1-2 \%$, owing to strong intermolecular interactions which cause the dominance of radiationless pathways in the process of decay of singlet excitations. ${ }^{12-14}$ According to quantum mechanical calculations, the photoluminescence of lightemitting oligomers is quenched when the long axes of adjacent conjugated chains are parallel to one another while a nonnegligible emission should be observed when an angle appears between the long axes of adjacent chains. ${ }^{15}$

So far, nothing has been reported concerning the light emission properties of thiophene oligomers modified by the introduction of a thienyl-S,S-dioxide unit, except the fact that some of these compounds display high photoluminescence efficiencies and electron affinities in the solid state and are worth studying as a new class of organic materials suitable for LED technology. 4

Photoluminescence is a very complex phenomenon, and full understanding of its behavior in such modified oligothiophenesin particular the importance of nonradiative pathways for energy relaxation, such as intersystem crossing or internal conversion, related to intrinsic molecular features - will require much more experimental work including detailed time-resolved measurements. Moreover, the conformational and electronic properties of isolated oligothiophene $S, S$-dioxides have just begun to be investigated $^{16}$ and much has still to be done to elucidate, for example, the contribution of oxygen lone pairs to excited-state orbitals. Thus, at the present state of knowledge, identification of guidelines for the synthesis of new molecules with high solidstate photoluminescence efficiencies has to rely on largely empirical criteria.

The data reported in this paper indicate that there is a correlation between packing modalities and photoluminescence efficiencies in oligothiophene $S, S$-dioxides 1-3. Assuming that the interpretation criteria for the solid-state photoluminescence properties of conventional oligothiophenes ${ }^{14,15}$ also hold for these modified oligomers, then the quite remarkable $45 \%$ photoluminescence efficiency of trimer 1 should be associated with the lack of alignment of molecular long axes in the crystalline packing (Figure 4, top), while the low $2 \%$ efficiency of heptamer $\mathbf{3}$ should be associated with the parallel arrangement of molecular long axes (Figure 4, bottom). The $12 \%$ efficiency of pentamer 2 should be viewed as being related to an intermediate type of packing between that of $\mathbf{1}$ and $\mathbf{3}$. In other words, as the packing modalities resemble more and more those of conventional oligomers, ${ }^{5,9,18}$ photoluminescence is progressively switched off. Heptamer $\mathbf{3}$ has a way of packing which

(14) (a) Theander, M.; Inganas, O.; Mammo, W.; Olinga, T.; Svensson, M.; Andersson, M. R. J. Phys. Chem. B. 1999, 103, 7771. (b) Benicori, T.; Bongiovanni, G.; Botta, C.; Cerullo, G.; Lanzani, G.; Mura, A.; Rossi, L.; Sannicolo', F.; Tubino, R. Phys. Rev. B 1998, 58, 9082. (c) Yang, M. Kuroda, Y. Shiraishi, T. Kobayashi. J. Chem. Phys. 1998, 109, 8442. (d) DiCésare, N.; Belletête, M.; Garcia, E. R.; Leclerc, M.; Durocher, G. J. Phys. Chem. A 1999, 103, 3864.

(15) (a) Beljonne, D.; Cornil, J.; Friend, R. H.; Jansen, R. A. J.; Brédas, J. L. J. Am. Chem. Soc. 1996, 118, 6453. (b) Brédas, J. L.; Cornil, J.; Beljonne, D.; Dos Santos, D. A.; Shuai. Z. Acc. Chem. Res. 1999, 32, 267.

(16) Barbarella, G.; Favaretto, L.; Sotgiu, G.; Zambianchi, M.; Arbizzani, C.; Bongini, A.; Mastragostino, M. Chem. Mater. 1999, 11, 2533.

(17) (a) Horowitz, G.; Bachet, B.; Yassar, A.; Lang, P.; Demanze, F.; Fave, J. L.; Garnier, F. Chem. Mater. 1995, 7, 1337. (b) Siegrist, T.; Fleming, R. M.; Haddon, R. C.; Laudise, R. A.; Lovinger, A. J.; Katz, H. E.; Bridenbaugh, P.; Davis, D. D. J. Mater. Res. 1995, 10, 2170. (c) Liao, J. H.; Benz, M.; LeGoff, E.; Kanatzidis, M. G. Adv. Mater. 1994, 6, 135.

(18) (a) Garnier, F.; Yassar, A.; Hajlaoui, R.; Horowitz, G.; Deloffre F.; Servet, B.; Ries, S.; Alnot, P. J. Am. Chem. Soc. 1993, 11, 15, 8716. (b) Garnier, F.; Hajlaoui, R.; El Kassmi, A.; Horowitz, G.; Laigre, L.; Porzio, W.; Armanini, M.; Provasoli, F. Chem. Mater. 1998, 10, 3334. (c) Laquindanum, J. G.; Katz, H. E.; Lovinger, A. J. J. Am. Chem. Soc. 1998, 120, 664. (d) Li, W.; Katz, H. E.; Lovinger, A. J.; Laquindanum, J. G. Chem. Mater. 1999, 11, 458. 
strongly resembles that of the best ordered thiophene oligomers reported so far ${ }^{5 \mathrm{c}}$ despite the presence of the oxygen atoms and the aliphatic chains. The molecule is rigorously planar, the aliphatic chains are symmetrically arranged as the wings of a flying bird, and the molecular planes are strictly parallel. In this way, photoluminescence is quenched. It is worth noting, in this respect, that the trend of photoluminescence efficiencies in 1-3 parallels that of the cohesion energy (which is greatest in the crystals of $\mathbf{3}$ ) and of $\mathrm{C}-\mathrm{H} \cdots \mathrm{O}$ intermolecular distances (which are the shortest in the heptamer, as shown in Table 1). Both parameters are indicative of how close the molecules are in the crystal and, the closer the molecules are, the greater is the importance of intermolecular interactions in furnishing nonradiative channels for energy relaxation from the excited state. In other words, the simultaneous occurrence of more planar conformations of the aromatic backbone and a tighter arrangement of the hexyl chains bring about a more cohesive packing of molecules $\mathbf{2}$ and $\mathbf{3}$ and leads to a greater number of nonradiative pathways. The values of radiative and nonradiative photoluminescence decay times $\left(\tau_{\mathrm{R}}\right.$ and $\tau_{\mathrm{NR}}$, reported in the Experimental Section) support this view.

The photoluminescence spectra of $\mathbf{1 - 3}$ in solution offer an interesting and complementary prospect of how much the light emission properties of these molecules depend on the type of environment. The photoluminescence spectra of $\mathbf{1 - 3}$ (see Figure 6) show a very weak light emission intensity for all the compounds in methylene chloride. However, when the solvent used is Decalin, less polar and more viscous than $\mathrm{CH}_{2} \mathrm{Cl}_{2}$, the photoluminescence intensity is increased by more than 1 order of magnitude for the longer oligomers and the emission bands display some fine structures. Interestingly, the emission frequencies of $\mathbf{1 - 3}$ in Decalin are blue shifted with respect to those in the solid state (Table 2). Likewise, heptamer $\mathbf{3}$ is no longer planar in solution and the delicate balance of inter- and intramolecular interactions (Table 1) at the origin of the coplanar conformation of this long and heavily substituted oligomer in the solid state is disrupted.

Turning to the solid-state characteristics of $\mathbf{1 - 3}$, to be able to design new molecules, it is of primary importance to obtain deeper insight into the nature of the specific inter- and intramolecular interactions on which solid-state conformation and molecular packing are built. As shown in Table 1, the hydrogen-bonding interactions between donor groups, oxygen atoms in particular, and acceptor neighboring $\mathrm{C}-\mathrm{H}$ groups as well as intramolecular $\mathrm{S} \cdots \mathrm{S}$ interactions are of main importance in 1-3. Apparently, the balance between all these interactions and the dipole-dipole intermolecular interactions due to the presence of $\mathrm{SO}_{2}$ groups defines conformation and packing. The introduction of the oxygen atoms disrupts the herringbone $/ \pi-\pi$ stacking duality generally found in the solid state for unsubstituted oligothiophenes. $5,9,17$

The interplay of all the forces in play is clearly shown in Figures $1-4$. On going from the trimer (one $\mathrm{SO}_{2}$ for two thienyl rings) to the heptamer (one $\mathrm{SO}_{2}$ for six thienyl rings), the progressively increasing importance of weak hydrogen-bonding and sulfur-sulfur interactions overwhelms the dipolar interactions and leads to progressively more planar conformations, greater packing energies, and alignment of molecular long axes. It is this balance of forces that makes the prediction of the solidstate arrangement (conformation and packing) of thiophenebased oligomers difficult. The "plasticity" of the thienyl rings ${ }^{5 b}$ allows for the maximum cooperation of all the forces in play and intra- and intermolecular interactions are set up in any possible way with only the limitation of steric constraints.

\section{Conclusion}

We have reported the first single-crystal X-ray structures of thiophene oligomers modified by the introduction of one thienyl$S, S$-dioxide unit and shown that the self-assembly modalities in single crystals depend on the relative number of conventional and modified thienyl units present in the molecule. When the oligomer is short, the packing is dominated by the dipolar intermolecular interactions associated with the presence of the sulfonyl groups and high photoluminescence efficiency is observed. On the contrary, when the packing is dominated by the intra- and intermolecular interactions associated with the presence of numerous unmodified thienyl rings, the photoluminescence efficiency drops to a few percent. The data confirm that the introduction of a thienyl-S,S-dioxide unit into the backbone of a thiophene oligomer is a useful strategy to orient the packing forces and to control the photoluminescence efficiency in the solid state.

On the whole, our data point out the extreme versatility of thiophene-based oligomers which can be viewed as a stable network of $\pi$-electrons that can be modified at will in order to optimize relevant properties, charge mobility, ${ }^{5 \mathrm{c}, 18}$ and photoluminescence efficiency, ${ }^{4 a}$ in particular. We believe that, in this respect, a challenging question to answer is whether it is possible to optimize both properties at the same time, in the same molecule, and in the same environment.

\section{Experimental Section}

Materials. The synthesis, the electrochemical characterization, and the chemical and electrochemical polymerization of $\mathbf{1 - 3}$ are reported in ref 16.

X-ray Crystallographic Studies. All X-ray measurements were carried out on a rotating-anode Siemens P4RA-M18X diffractometer by using graphite-monochromated Mo K $\alpha$ radiation $(\lambda=0.71069 \AA)$. In all cases, intensity data were collected in the $\theta-2 \theta$ scan mode. All intensities were corrected for Lorentz and polarization effects, but not for absorption in view of the low absorption coefficients and small crystal dimensions.

All structures were solved by direct methods (SHELX86 program) and were refined through full-matrix least-squares calculations based on $F^{2}$ (SHEXL93) for all unique reflections. ${ }^{19,20}$ In all the structures, after isotropic refinements of non-H atoms, anomalously high or low thermal parameters, unrealistic bond distances, and relevant residuals in $\Delta F$ maps provided evidence of statistical disorders involving, in all cases, one outer thiophene ring and the fifth $\mathrm{C}$ atom of one hexyl chain. The disordered thiophene rings assumes two opposite orientations (anti or syn) which arises from a rotation of $\sim 180^{\circ}$ around the $\mathrm{C}-\mathrm{C}$ interring bond. In disordered hexyl chains, one $\mathrm{C}$ atom (always the fifth) was found to be distributed over at least two alternative sites, but some residuals in $\Delta F$ maps could be consistent with more complex disorder models. For all disordered thiophene rings, it was deemed necessary to apply bond length constraints to atoms with either major or with minor site occupancy factors; the following values were set fixed: all $\mathrm{C}-\mathrm{S}=1.70 \AA, \mathrm{C} 2-\mathrm{C} 3$ and $\mathrm{C} 4-\mathrm{C} 5=1.35 \AA$, and $\mathrm{C} 3-\mathrm{C} 4=1.42 \AA$. In disordered moieties of hexyl chains of compounds T3 and T5, a $\mathrm{C}-\mathrm{C}$ bond length constraint of $1.50 \AA$ was applied. In all the structures, the hydrogen atoms were placed in calculated positions at a bond distance of $0.93 \AA$ for those bonded to thienyl C or from 0.96 to 0.98 $\AA$ for those bonded to alkyl C. Their thermal parameters were set 1.2 times $U_{\text {eq }}$ of the bonded atoms.

Compound 1. $\mathrm{C}_{24} \mathrm{H}_{32} \mathrm{O}_{2} \mathrm{~S}_{3}$; orange yellow, air-stable crystals, grown by slow evaporation of chloroform solutions. The one selected had approximate dimensions of $0.33 \times 0.28 \times 0.25 \mathrm{~mm}$. All measurements were carried out at room temperature with X-ray source power of 52 $\mathrm{kV}$ and $110 \mathrm{~mA}$. Unit cell dimensions were obtained from least-squares

(19) Sheldrick, G. M. Acta Crystallogr. Sect. A 1990, 46, 467.

(20) Sheldrick, G. M. SHELXL93. Program for the refinement of crystal structures. University of Göttingen, Germany, 1993. 
fit to the setting angles of 35 automatically centered reflections in the $5-14^{\circ} \theta$ range. Crystals were orthorhombic, space group $P 22_{1} 2_{1} 2_{1}$, with $a=9.552(2) \AA, b=14.509$ (3) $\AA$, and $c=17.781(3) \AA$ and $V=2464.2$ (8) $\AA^{3}$. For $Z=4$, the calculated density was $1.209 \mathrm{Mg} / \mathrm{m}^{3}$. A total of 3154 reflections were collected in the $1.8-25.0^{\circ} \theta$ range. Absorption correction was not applied $\left(\mu=0.318 \mathrm{~mm}^{-1}\right)$ to intensities. Data were averaged to 2961 unique reflections $\left(R_{\text {int }}=0.0275\right)$, of which 1634 had $I \geq 2 \sigma(I)$. All non-hydrogen atoms were refined anisotropically, except the disorder $\mathrm{C}$ atoms, whose isotropic $U$ were constrained to be equal for atoms with alternative occupancy factors. Least-squares refinement of 266 parameters with 18 restraints led to final $R$ and $w R 2$ values of 0.0664 and 0.1591 for reflections with $I \geq 2 \sigma(I)$ and of 0.1354 and 0.1856 for all reflections. The weighting scheme used throughout was $w=1 /\left[\sigma^{2}\left(F_{\mathbf{0}}{ }^{2}\right)+(0.1050 P)^{2}\right]$, where $P=\left(F_{\mathbf{0}}{ }^{2}+2 F_{\mathbf{c}}{ }^{2}\right) / 3$, and the final goodness of fit on $F^{2}$ was 0.991 . The occupancy factors for the disordered thiophene ring atoms are $0.516(7)$ for the anti orientation and 0.484(7) for the syn one, whereas in the disordered hexyl chain, C(45) was split into two sites with occupancy 0.65(1) and 0.35(1), respectively. Refinement of the Flack absolute structure parameter $c$ led to a final value of $0.0(2)$, so that the crystal chirality appeared to be assigned correctly. ${ }^{21}$ The largest difference peak and hole were 0.254 and $-0.265 \AA^{3}$, respectively.

Compound 2. $\mathrm{C}_{32} \mathrm{H}_{36} \mathrm{O}_{2} \mathrm{~S}_{5}$; red, air-stable crystals, grown by slow evaporation of 2-propanol solutions. The one selected had approximate dimensions $0.25 \times 0.22 \times 0.18 \mathrm{~mm}$. All measurements were carried out at 200(1) K with X-ray source power of $52 \mathrm{kV}$ and $130 \mathrm{~mA} .{ }^{22}$ Unit cell dimensions were obtained from least-squares fit to the setting angles of 35 automatically centered reflections in the $4-16^{\circ} \theta$ range. Crystals are monoclinicic, space group $P 2{ }_{1} / n$, with $a=15.287(2) \AA$, $b=8.518(3) \AA$, and $c=23.425(3) \AA, \beta=90.62(1)^{\circ}$, and $\mathrm{V}=3050.0$ (11) $\AA^{3}$. For $Z=4$, the calculated density was $1.335 \mathrm{Mg} / \mathrm{m}^{3}$. A total of 5708 reflections were collected in the $2.5-24.0^{\circ} \theta$ range. Absorption correction was not applied $\left(\mu=0.409 \mathrm{~mm}^{-1}\right)$. Data were averaged to 4725 unique reflections $\left(R_{\text {int }}=0.0417\right)$, of which 2842 have $I \geq$ $2 \sigma(I)$.

All non-hydrogen atoms with full occupancy were refined anisotropically, along with the disordered $\mathrm{S}(1 \mathrm{E})$ and $\mathrm{C}(35)$ at major sites; other disordered non-H atoms were refined isotropicaly. Least-squares refinement of 375 parameters with 14 restraints led to final $R$ and $w R 2$ values of 0.0797 and 0.2057 for reflections with $I \geq 2 \sigma(I)$, and of 0.1382 and 0.2346 for all reflections. The weighting scheme used throughout was $w=1 /\left[\sigma^{2}\left(F_{0}^{2}\right)+(0.1325 P)^{2}\right]$, where $P=\left(F_{\mathrm{o}}{ }^{2}+2 F_{\mathrm{c}}{ }^{2}\right) / 3$, and the final goodness of fit on $F^{2}$ was 1.044. The occupancy factors for the disordered thiophene ring atoms were $0.662(8)$ for the anti orientation and $0.338(8)$ for the syn one, whereas in the disordered hexyl chain, $\mathrm{C}(35)$ is split into two sites with occupancy $0.78(2)$ and $0.22(2)$, respectively. The largest difference peak and hole were 0.662 and $-0.392 \AA^{3}$, respectively.

Compound 3. $\mathrm{C}_{40} \mathrm{H}_{40} \mathrm{O}_{2} \mathrm{~S}_{7}$; deep red, air-stable crystals, grown by slow evaporation of 2-propanol/trichloroethylene solutions. The one selected had approximate dimensions of $0.28 \times 0.24 \times 0.19 \mathrm{~mm}$. All measurements were carried out at 200(1) K with X-ray source power of $52 \mathrm{kV}$ and $130 \mathrm{~mA}$. Unit cell dimensions were obtained from leastsquares fit to the setting angles of 33 automatically centered reflections in the $5-17^{\circ} \theta$ range. Crystals were triclinicic, space group $P \overline{1}$, with $a=10.614(2) \AA, b=13.133(2) \AA$, and $c=15.427(3), \alpha=96.67(2)^{\circ}$, $b=109.20(2)^{\circ}$, and $\gamma=107.43(2)^{\circ}$ and $V=1881.6(5) \AA^{3}$. For $Z=$ 2 , the calculated density was $1.372 \mathrm{Mg} / \mathrm{m}^{3}$. A total of 7687 reflections were collected in the $1.9-25.0^{\circ} \theta_{-}$range. Absorption correction was

(21) Flack, H. D. Acta Crystallogr. Sect. A 1983, 39, 876.

(22) The structure of pentamer $\mathbf{2}$ was previously determined at room temperature by using another crystal. Cell dimensions found at $293 \mathrm{~K}$ were as follows: $a=15.409(2) \AA, b=8.569(3) \AA$, and $c=23.769$ (3) $\AA, \beta=$ 92.11(1) ${ }^{\circ}$, and $V=3136.2(11) \AA^{3}$. A relevant change $\left(-1.49^{\circ}\right)$ of the $\beta$-angle on passing at low temperature should be noted. The quality of the first determination is slightly lower than that at $200 \mathrm{~K}$, and, as expected, is characterized by higher thermal motion parameters, in particular for the alkyl $\mathrm{C}$ atoms. Furthermore, the same type of atomic disorder (roughly to the same extent) was found to be present also in the first crystal. not applied $\left(\mu=0.454 \mathrm{~mm}^{-1}\right)$. Data were averaged to 6529 unique reflections $\left(R_{\text {int }}=0.0356\right)$, of which 5033 had $I \geq 2 \sigma(I)$. All nonhydrogen atoms were refined anisotropically, except those of the disordered thiophene ring with lower site occupancy which were treated isotropically with a common temperature factor. Least-squares refinement of 454 parameters with 10 restraints led to final $R$ and $w R 2$ values of 0.0792 and 0.2126 for reflections with $I \geq 2 \sigma(I)$, and of 0.0970 and 0.2293 for all reflections. The weighting scheme used throughout was $w=1 /\left[\sigma^{2}\left(F_{\mathrm{o}}{ }^{2}\right)+(0.1257 P)^{2}+1.8893 P\right]$, where $P=\left(F_{\mathrm{o}}{ }^{2}+2 F_{\mathrm{c}}{ }^{2}\right) / 3$, and the final goodness of fit on $F^{2}$ was 1.044 . The occupancy factors for the disordered thiophene ring atoms were $0.738(8)$ for the syn orientation and $0.262(8)$ for the anti one, whereas in the disordered hexyl chain, $\mathrm{C}(45)$ was split into two sites with occupancy 0.64(2) and $0.36(2)$, respectively. The largest difference peak and hole were 1.508 and -1.179 e. $\AA^{3}$, respectively.

Packing potential energy calculations of $\mathbf{1}-\mathbf{3}$ were carried out using a semiempirical approach ${ }^{8 a}$ based on an atom-atom potential energy method. ${ }^{8 \mathrm{~b}}$ For each structure, four possible ordered crystals were considered, i.e., combining the two orientations of terminal thiophene with the two conformations of one hexyl chain. The PC and ppe values in Table 1 were averaged over those obtained for the corresponding ordered crystals. $\mathrm{C}-\mathrm{H} \cdots$ and $\mathrm{C}-\mathrm{H} \cdots$ contacts were calculated with $\mathrm{C}-\mathrm{H}$ distances normalized to their neutron value $(1.08 \AA)$.

${ }^{13}$ C CP-MAS NMR. The spectra were obtained using a spectrometer operating at $75.45 \mathrm{MHz}$ for carbon-13. The solid samples were finely grounded, carefully packed into a $7-\mathrm{mm}$ zirconia rotor, and spun at the magic angle at a rate of $3.8-4.2 \mathrm{kHz}$ as required to reduce the overlapping between signals and spinning sidebands. The ${ }^{1} \mathrm{H}$ pulse duration was $4 \mu \mathrm{s}$, which was also the value for ${ }^{13} \mathrm{C}$ as required by the Hartmann-Hahn condition. The recycling delay was $10 \mathrm{~s}$, whereas the contact time chosen for the cross-polarization was $1.5 \mathrm{~ms}$. The number of transients required to achieve good signal-to-noise ratios varied with the cicumstances but was tipically 2000-8000. The signal assignment was made with the aid of the nonquaternary suppression experiments for which a dipolar dephasing of the order $40 \mu$ s was required in order to cancel the signals of the hydrogenated carbons. ${ }^{23 a, b}$ The chemical shifts were measured for the adamantane, as reference, and scaled to TMS using the value of $29.4 \mathrm{ppm}$ for the lower frequency signal of the adamantane.

Photoluminescence Measurements. PL efficiency measurements of the microcrystalline powders of $\mathbf{1 - 3}$ were performed by means of a 4-in. integrative sphere (Labsphere) ${ }^{24}$ by exciting the samples with an $\mathrm{Ar}$ ion laser $\left(\lambda_{\mathrm{ex}}=363 \mathrm{~nm}\right)$ and collecting the PL with CCD spectrograph (Ocean Optics). To prevent photooxidation, the measurements were carried out under dry nitrogen. Absorption and photoluminescence spectra were recorded, respectively, with a Perkin-Elmer $\lambda 5$ spectrophotometer and with a Spex Fluorolog II spectrofluorometer equipped with continuous 150-W Xe lamp and a Hamamatsu R-9928 or R-3896 photomultiplier tube. Solutions were $10^{-5} \mathrm{M}$ in methylene chloride and Decalin. The solvents were purchased from Baker Analyze and were spectroscopic grade. Decalin was further purified by silicagel chromatography.

Acknowledgment. We are grateful to MURST ("Electrochemical and Electronic Devices with Polymer Components") for financial support.

Supporting Information Available: Tables of crystal data and structure refinements, positional and thermal parameters, bond distances and angles, torsion angles, shortest intermolecular distances, selected least-squares planes, drawings of the disorder models and molecular packing for $\mathbf{1 - 3}$ (PDF). This material is available free of charge via the Internet at http://pubs.acs.org.

\section{JA000834H}

(23) (a) Voelkel, R. Angew. Chem., Int. Ed. Engl. 1988, 27, 1468. (b) Opella, S. J.; Frey, M. H. J. Am. Chem. Soc. 1979, 101, 5854.

(24) De Mello, J. C.; Wittmann, H. F.; Friend. R. H. Adv. Mater. 1997 $9,230$. 